\title{
Crystal structure of chlorotris(2,2,6,6-tetramethyl-3,5-heptanedionato)- zirconium(IV), $\left[\mathrm{ZrCl}\left\{\left(\mathrm{CH}_{3}\right)_{3} \mathrm{CCOCHCOC}\left(\mathrm{CH}_{3}\right)_{3}\right\}_{3}\right]$
}

\author{
U. Casellato \\ C.N.R. - ICTIMA. Corso Stati Uniti, 4. I-35100 Padova. Italy \\ D. Fregona and R. Graziani
}

Università di Padova, Dipartimento di Chimica Inorganica, Via Loredan, 4, I-35131 Padova, Italy

Received September 26, 1996, CSD-No. 402641

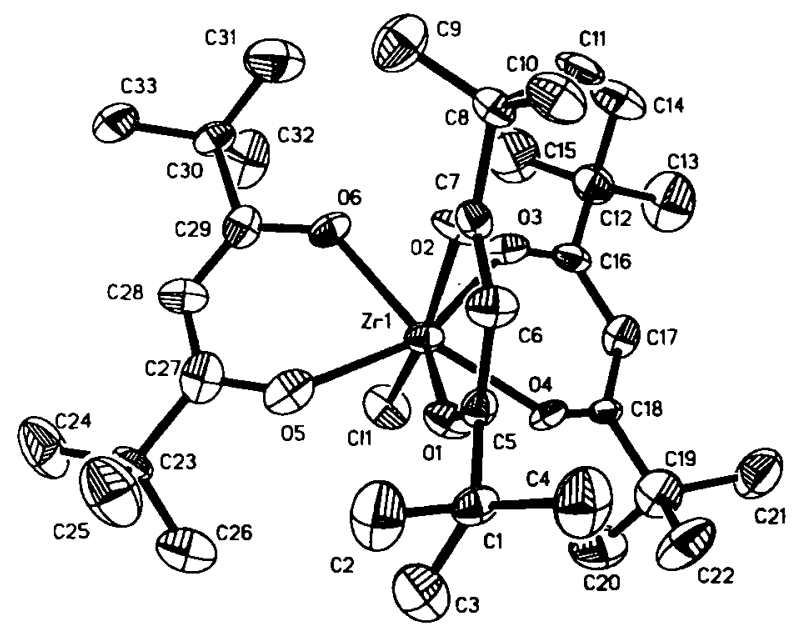

Source of material: The title compound was obtained by reacting 2,2,6,6-tetramethyl-3,5-heptanedione with $\mathrm{ZrCl}_{4}$ (molar ratio $1: 4$ ) in ethanol. White crystals separated from the mother solution on standing.

Methyl $\mathrm{H}$ atoms were not introduced in the calculations. One chlorine atom and three bidentate ligands are directly bonded to the $\mathrm{Zr}$ atom forming an irregular coordination polyhedron. $\mathrm{Zr}-\mathrm{O}$ bonds range from $2.09 \AA$ to $2.15 \AA$; the $\mathrm{Zr}-\mathrm{Cl}$ distance is $2.496(3) \AA$.
$\mathrm{C}_{33} \mathrm{H}_{57} \mathrm{ClO}_{6} \mathrm{Zr}$, monoclinic, $P 2_{1}$ (No. 4), $a=10.679(5) \AA$, $b=18.263(7) \AA, c=10.973(5) \AA, \beta=117.68(5)^{\circ}, V=1895.2 \AA^{3}, Z=2$, $R(F)=0.049, R_{\mathrm{w}}(F)=0.049$.

Table 1. Parameters used for the $\mathrm{X}$-ray data collection

$\begin{array}{ll}\text { Crystal: } & \text { white prism, max. dim. } 0.2 \mathrm{~mm} \\ \text { Wavelength: } & \text { Mo } K_{\alpha} \text { radiation }(0.71073 \AA) \\ \mu: & 261.00 \mathrm{~cm}^{-1} \\ \text { Diffractometer: } & \text { Philips } \\ \text { Scan mode: } & 2 \theta \\ T_{\text {measurement: }} & 293 \mathrm{~K} \\ 2 \theta_{\text {max }} & 54^{\circ} \\ \text { N( } h k)_{\text {unique: }} & 2524 \\ \text { Criterion for } F_{\mathrm{o}}: & F_{\mathrm{o}}>3 \sigma\left(F_{\mathrm{o}}\right) \\ \text { N(param) } & 379 \\ \text { Program: } & \text { SHELXX-76 }\end{array}$

Table 2. Final atomic coordinates and displacement parameters (in $\AA^{2}$ )

\begin{tabular}{llllll}
\hline Atom & Site & $x$ & $y$ & $z$ & $U_{\text {iso }}$ \\
\hline $\mathrm{H}(6)$ & $2 a$ & $1.169(1)$ & $0.483(1)$ & $0.828(1)$ & 0.07 \\
$\mathrm{H}(17)$ & $2 a$ & $0.699(1)$ & $0.709(1)$ & $0.253(1)$ & 0.07 \\
$\mathrm{H}(28)$ & $2 a$ & $0.711(1)$ & $0.256(1)$ & $0.237(1)$ & 0.07 \\
\hline
\end{tabular}

Table 3. Final atomic coordinates and displacement parameters (in $\dot{\AA}^{2}$ )

\begin{tabular}{|c|c|c|c|c|c|c|c|c|c|c|}
\hline Atom & Site & $x$ & $y$ & $z$ & $U_{11}$ & $U_{22}$ & $U_{33}$ & $U_{12}$ & $U_{13}$ & $U_{23}$ \\
\hline $\operatorname{Zr}(1)$ & $2 a$ & $0.8482(1)$ & 0.4593 & $0.3747(1)$ & $0.0333(3)$ & $0.0565(4)$ & $0.0348(3)$ & $-0.0017(8)$ & $0.0149(2)$ & $0.0001(8)$ \\
\hline $\mathrm{Cl}(1)$ & $2 a$ & $0.7498(2)$ & $0.4611(5)$ & $0.1192(2)$ & $0.0719(8)$ & $0.0904(9)$ & $0.0346(7)$ & $0.0147(9)$ & $0.0152(6)$ & $-0.0035(9)$ \\
\hline$O(1)$ & $2 a$ & $1.0691(4)$ & $0.4570(7)$ & $0.5131(4)$ & $0.0365(9)$ & $0.0781(9)$ & $0.0362(9)$ & $-0.007(1)$ & $0.0136(8)$ & $-0.008(1)$ \\
\hline$O(2)$ & $2 a$ & $0.8539(4)$ & $0.4600(8)$ & $0.5706(4)$ & $0.0435(9)$ & $0.0984(9)$ & $0.0366(9)$ & $0.012(1)$ & $0.0229(8)$ & $0.010(1)$ \\
\hline$O(3)$ & $2 a$ & $0.6782(7)$ & $0.5325(5)$ & $0.3425(6)$ & $0.0551(9)$ & $0.061(1)$ & $0.0604(9)$ & $0.0004(9)$ & $0.0470(9)$ & $0.0055(9)$ \\
\hline $\mathrm{O}(4)$ & $2 a$ & $0.9253(6)$ & $0.5637(5)$ & $0.3496(6)$ & $0.0375(9)$ & $0.0371(9)$ & $0.0543(9)$ & $-0.0096(9)$ & $0.0295(9)$ & $0.0017(9)$ \\
\hline$O(5)$ & $2 a$ & $0.9210(7)$ & $0.3563(6)$ & $0.3542(7)$ & $0.0505(9)$ & $0.086(1)$ & $0.0508(9)$ & $-0.0139(9)$ & $0.0064(9)$ & $0.001(1)$ \\
\hline$O(6)$ & $2 a$ & $0.6797(7)$ & $0.3855(6)$ & $0.3434(7)$ & $0.0353(9)$ & $0.067(1)$ & $0.0609(9)$ & $-0.0147(9)$ & $0.0075(9)$ & $-0.0060(9)$ \\
\hline$C(1)$ & $2 a$ & $1.3069(6)$ & $0.4616(8)$ & $0.6871(6)$ & $0.0312(9)$ & $0.068(1)$ & $0.0475(9)$ & $-0.008(1)$ & $0.0120(9)$ & $-0.001(1)$ \\
\hline $\mathrm{C}(2)$ & $2 a$ & $1.3556(8)$ & $0.3836(4)$ & $0.7210(8)$ & $0.048(1)$ & $0.099(1)$ & $0.188(1)$ & $0.011(1)$ & $0.036(1)$ & $0.002(1)$ \\
\hline$C(3)$ & $2 a$ & $1.3349(8)$ & $0.4888(5)$ & $0.5742(6)$ & $0.061(1)$ & $0.209(1)$ & $0.102(1)$ & $-0.021(1)$ & $0.037(1)$ & $0.048(1)$ \\
\hline$C(4)$ & $2 a$ & $1.3893(8)$ & $0.5079(5)$ & $0.8148(6)$ & $0.048(1)$ & $0.175(1)$ & $0.109(1)$ & $-0.038(1)$ & $0.010(1)$ & $-0.075(1)$ \\
\hline $\mathrm{C}(5)$ & $2 a$ & $1.1497(6)$ & $0.4627(8)$ & $0.640616)$ & $0.0321(9)$ & $0.0480(9)$ & $0.0443(9)$ & $-0.012(1)$ & $0.0130(9)$ & $-0.012(1)$ \\
\hline $\mathrm{C}(6)$ & $2 a$ & $1.0969(6)$ & $0.4591(9)$ & $0.7373(6)$ & $0.0411(9)$ & $0.085(1)$ & $0.0386(9)$ & $-0.012(1)$ & $0.0129(9)$ & $-0.003(1)$ \\
\hline
\end{tabular}


Table 3. (Continued)

\begin{tabular}{|c|c|c|c|c|c|c|c|c|c|c|}
\hline Atom & Site & $x$ & $y$ & $z$ & $U_{11}$ & $U_{22}$ & $U_{33}$ & $U_{12}$ & $U_{13}$ & $U_{23}$ \\
\hline$C(7)$ & $2 a$ & $0.9524(6)$ & $0.4564(8)$ & $0.6988(6)$ & $0.0367(9)$ & $0.065(1)$ & $0.0409(9)$ & $0.015(1)$ & $0.0128(9)$ & $-0.017(1)$ \\
\hline$C(8)$ & $2 a$ & $0.8934(6)$ & $0.4540(7)$ & $0.8012(6)$ & $0.0510(9)$ & $0.069(1)$ & $0.0399(9)$ & $0.006(1)$ & $0.0253(9)$ & $-0.005(1)$ \\
\hline$C(9)$ & $2 a$ & $0.8029(9)$ & $0.3854(7)$ & $0.7803(8)$ & $0.106(1)$ & $0.096(1)$ & $0.078(1)$ & $-0.036(1)$ & $0.025(1)$ & $0.007(1)$ \\
\hline$C(10)$ & $2 a$ & $1.0141(7)$ & $0.4695(8)$ & $0.9509(6)$ & $0.073(1)$ & $0.132(1)$ & $0.0385(9)$ & $0.029(1)$ & $0.0239(9)$ & $-0.009(1)$ \\
\hline$C(11)$ & $2 a$ & $0.7965(7)$ & $0.5228(6)$ & $0.7684(7)$ & $0.0853(9)$ & $0.076(1)$ & $0.0790(9)$ & $0.013(1)$ & $0.0745(9)$ & $0.004(1)$ \\
\hline$C(12)$ & $2 a$ & $0.5089(8)$ & $0.6190(7)$ & $0.2759(8)$ & $0.061(1)$ & $0.065(1)$ & $0.071(1)$ & $0.012(1)$ & $0.0290(9)$ & $0.002(1)$ \\
\hline$C(14)$ & $2 a$ & $0.5000(8)$ & $0.6170(7)$ & $0.4122(8)$ & $0.062(1)$ & $0.151(1)$ & $0.099(1)$ & $0.026(1)$ & $0.0520(9)$ & $-0.007(1)$ \\
\hline$C(15)$ & $2 a$ & $0.3828(8)$ & $0.5677(8)$ & $0.1694(9)$ & $0.068(1)$ & $0.145(1)$ & $0.149(1)$ & $0.000(1)$ & $0.032(1)$ & $-0.044(1)$ \\
\hline$C(16)$ & $2 a$ & $0.6423(8)$ & $0.5950(7)$ & $0.2938(8)$ & $0.038(1)$ & $0.067(1)$ & $0.051(1)$ & $-0.009(1)$ & $0.0090(9)$ & $-0.018(1)$ \\
\hline$C(17)$ & $2 a$ & $0.7310(8)$ & $0.6410(6)$ & $0.2634(8)$ & $0.055(1)$ & $0.044(1)$ & $0.061(1)$ & $0.007(1)$ & $0.012(1)$ & $0.012(1)$ \\
\hline$C(18)$ & $2 a$ & $0.8617(7)$ & $0.6211(5)$ & $0.2874(7)$ & $0.0382(9)$ & $0.036(1)$ & $0.0414(9)$ & $-0.0107(9)$ & $0.0299(9)$ & $-0.0093(9)$ \\
\hline$C(19)$ & $2 a$ & $0.9497(8)$ & $0.6765(7)$ & $0.2521(8)$ & $0.073(1)$ & $0.079(1)$ & $0.079(1)$ & $0.024(1)$ & $0.037(1)$ & $0.019(1)$ \\
\hline$C(20)$ & $2 a$ & $0.9338(8)$ & $0.6435(8)$ & $0.1168(8)$ & $0.149(1)$ & $0.165(1)$ & $0.105(1)$ & $-0.029(1)$ & $0.093(1)$ & $0.013(1)$ \\
\hline$C(23)$ & $2 a$ & $0.9633(7)$ & $0.2491(6)$ & $0.2554(8)$ & $0.0450(9)$ & $0.053(1)$ & $0.078(1)$ & $0.012(1)$ & $0.0342(9)$ & $-0.011(1)$ \\
\hline$C(24)$ & $2 a$ & $0.8831(8)$ & $0.1793(7)$ & $0.1673(8)$ & $0.078(1)$ & $0.126(1)$ & $0.142(1)$ & $-0.018(1)$ & $0.037(1)$ & $-0.093(1)$ \\
\hline$C(25)$ & $2 a$ & $1.0913(8)$ & $0.2275(7)$ & $0.3715(8)$ & $0.091(1)$ & $0.151(1)$ & $0.088(1)$ & $0.056(1)$ & $0.005(1)$ & $-0.034(1)$ \\
\hline$C(26)$ & $2 a$ & $1.0143(8)$ & $0.2959(7)$ & $0.1688(8)$ & $0.116(1)$ & $0.155(1)$ & $0.114(1)$ & $0.033(1)$ & $0.081(1)$ & $0.010(1)$ \\
\hline$C(27)$ & $2 a$ & $0.8739(8)$ & $0.2912(7)$ & $0.3002(8)$ & $0.046(1)$ & $0.065(1)$ & $0.056(1)$ & $-0.008(1)$ & $-0.010(1)$ & $0.002(1)$ \\
\hline$C(28)$ & $2 a$ & $0.7274(8)$ & $0.2773(7)$ & $0.2652(8)$ & $0.055(1)$ & $0.089(1)$ & $0.077(1)$ & $-0.009(1)$ & $0.0412(9)$ & $-0.004(1)$ \\
\hline$C(29)$ & $2 a$ & $0.6457(7)$ & $0.3205(6)$ & $0.2966(7)$ & $0.0441(9)$ & $0.052(1)$ & $0.0347(9)$ & $-0.0176(9)$ & $0.0222(9)$ & $0.0071(9)$ \\
\hline$C(30)$ & $2 a$ & $0.4903(7)$ & $0.2969(6)$ & $0.2667(8)$ & $0.0199(9)$ & $0.064(1)$ & $0.059(1)$ & $-0.0104(9)$ & $0.0128(9)$ & $-0.002(1)$ \\
\hline$C(31)$ & $2 a$ & $0.4643(8)$ & $0.3301(8)$ & $0.3829(8)$ & $0.086(1)$ & $0.152(1)$ & $0.120(1)$ & $-0.002(1)$ & $0.064(1)$ & $-0.021(1)$ \\
\hline$C(32)$ & $2 a$ & $0.3974(8)$ & $0.3379(7)$ & $0.1326(9)$ & $0.041(1)$ & $0.109(1)$ & $0.121(1)$ & $0.007(1)$ & $-0.014(1)$ & $0.029(1)$ \\
\hline$C(33)$ & $2 a$ & $0.4784(8)$ & $0.2171(6)$ & $0.2577(8)$ & $0.073(1)$ & $0.078(1)$ & $0.103(1)$ & $-0.037(1)$ & $0.0528(9)$ & $-0.024(1)$ \\
\hline
\end{tabular}

\section{References}

1. VonDreele, R. B.; Stezowski, J. J.; Fay, R. C: Crystal and molecular structure of chlorotris(acetylacetonato)zirconium(IV). J. Am. Chem. Soc. 93 (1971) 2887-2892.

2. Kyung Chun, H.; Steffen, W. L.; Fay, R. C.: Effects of crystal packing on the coordination geometry of eight-coordinate metal chelates. Crystal and molecular structure of tetrakis(1,3-diphenyl-1,3-propanedionato) zirconium(IV). Inorg. Chem. 18 (1979) 2458-2465

3. Clegg, W.: Redetermination of the structure of tetrakis(acetylacetonato)zirconium(IV). Acta Crystallogr. C43 (1987) 789-791.

4. Sheldrick, G. M: SHELX-76, Programs for Crystal Structure Determination. University of Cambridge, UK 1976. 